\title{
Regulatory effect of daphnetin on the balance of Th17 and Treg cells in the peripheral blood mononuclear cells from patients with unexplained recurrent pregnancy loss
}

\author{
ZHIQIN ZHANG ${ }^{1}$, SHENGGEN LONG ${ }^{2}$ ZHIHUI HUANG ${ }^{3}$, JUN TAN $^{2}$, \\ QIONGFANG WU', OUPING HUANG
}

${ }^{1}$ Department of Human Assisted Reproductive, Maternal and Child Health Affiliated Hospital of Nanchang University, Nanchang City, Jiangxi, PR China

${ }^{2}$ Department of Human Assisted Reproductive, Jiangxi Maternity and Child Healthcare Hospital, Nanchang City, Jiangxi, PR China ${ }_{3}^{3}$ Jiangxi Maternity and Child Healthcare Hospital, Nanchang City, Jiangxi, PR China

${ }^{4}$ Department of Gynecology, Jiangxi Maternity and Child Healthcare Hospital, Nanchang City, Jiangxi, PR China

\begin{abstract}
Introduction: There has a close relationship between the balance of Thelper 17 (Th17) cells and Foxp3+ regulatory T cells (Treg) and unexplained recurrent pregnancy loss (URPL). The present study is to investigate the regulatory effect of daphnetin, which is extracted from Daphne odora Var, on the balance of Th17 and Treg cells in the peripheral blood mononuclear cells (PBMCs) from patients with URPL.

Material and methods: PBMCs were isolated from 35 pregnant women with URPL and 35 women with normal early pregnancies, respectively and treated with daphnetin for three days. Flow cytometry was performed to measure the proportions of Th17 and Treg cells. The level of expression of IL-6, $T G F-\beta 1$ and IL-2 were detected using enzyme-linked immunosorbent assay (ELISA) and the level of expression of FoxP3, ROR t, signal transducers and activators of transcriotion 3 (STAT3) and STAT5 were detected by $R T-P C R$.

Results: The concentrations of Th17-type cytokines IL-2 were significantly decreased in the URPL group after treatment $(p<0.01)$. Treg-type cytokines such as TGF- $\beta 1$ and IL-6 were significantly increased after treatment $(p<0.01)$. At the same time, daphnetin may induce a decrease in the ratio of ROR $\gamma$ t to Foxp3 and a Treg cell bias, which would be beneficial for pregnancy maintenance. Futhermore the expression level of STAT3 were higher in the URPL patients whereas STAT5 were lower than those in the control subjects.

Conclusions: In conclusion, daphnetin may have regulatory effect on the balance of Th17 and Treg cells in the peripheral blood mononuclear cells from patients with URPL.
\end{abstract}

Key words: daphnetin, unexplained recurrent pregnancy loss, Th17 cells, Treg cells, peripheral blood mononuclear cells.

(Centr Eur J Immunol 2020; 45 (4): 403-408)

\section{Introduction}

Pregnancy loss is defined as the loss of a pregnancy prior to 24 weeks gestation. Recurrent pregnancy loss (RPL) has previously been defined as three or more pregnancy losses [1]. However, more recent guidelines have amended this definition to two or more pregnancy losses $[2,3]$. Unexplained recurrent pregnancy loss (URPL) affects approximately $50 \%$ of RPL patients, and it is largely associated with the failure of fetal-maternal immunologic tolerance [4]. In recent years, multiple studies have found that immune imbalance at the maternal-fetal interface plays a role in the pathogenesis of URPL, and interactions among an array of cytokines are believed to contribute to the ability of the maternal immune system to tolerate the genetically incompatible fetus $[5,6]$.

The related pathogenesis of URPL remains unknown [7]. However, it has been suggested that cytokines produced by $\mathrm{CD}^{+} \mathrm{T}$ cells may play an important role in the cause of URPL [8]. The CD4 ${ }^{+} \mathrm{T}$ cells can be classified into the following subsets: $\mathrm{T}$ helper (Th)1, Th2, Th17 and regula-

Correspondence: Ouping Huang, Department of Gynecology, Jiangxi Maternity and Child Healthcare Hospital, No. 381 Ba-yi Rd, Nanchang City 330006, Jiangxi, PR China, e-mail: wangfenghop@ 126.com Submitted: 28.05.2020; Accepted: 19.06.2020 
tory $\mathrm{T}$ (Treg) cells according to their functions. Several studies have reported that the altered immunity in URPL is dominated by the Th1/Th2 hypothesis. They found that the Th1/Th2 ratio were higher in URPL patients compared to normal pregnant controls, which indicates that the changes in the proportion of Th1 and Th2 may be related to occurrence of URPL $[9,10]$. However, the emerging concept of the Th17/Treg balance has challenged the conventional paradigm of Th1/Th2 hypothesis. Accumulating evidence suggests that Th17 and Treg cells play the antagonist roles on the URPL [11-14], they proposed the increase of the Th17/Treg ratio as a cause for URPL.

At present, there is no specific remedy available in clinic for curing URPL. Daphnetin (7,8-fihydroxycoumarin) extracting from Daphne odora var. marginata (D. marginata) mainly contains coumarin compounds. Daphnetin has been used to treat various autoimmune diseases such as rheumatoid arthritis possibly through modulating the balance of Tregs and Th17 [16]. However, the therapeutic potential of daphnetin on URPL is still unclear. Therefore, in this study, we firstly investigated the roles of the balance between Th17 and Treg in the development of URPL in order to dissect the mechanisms of daphnetin in terms of URPL therapeutics.

\section{Material and methods}

\section{Daphnetin}

Daphnetin was purchased from Tauto Biotech Co., Ltd (Shanghai, China), and the purity was at least $99.4 \%$.

\section{Subjects}

The study enrolled 35 URPL patients with a mean age of 30.55 years (range: $22-43$ years) who had at least three consecutive spontaneous miscarriages that occurred before 20 weeks gestation and had no chromosomal, anatomic, endocrine dysfunctions, or infections of the reproductive tract. All male partners had normal semen status, according to criteria from the World Health Organization. The control group comprised 35 non-pregnant healthy women with a mean age of 31.25 years (range: $23-44$ years) who at least one successful pregnancy without any disease. Control group women had no history of any stillbirths, preterm and post-term labor, ectopic pregnancy. All the subjects were patients of the department of Obstetrics and Gynecology at the Jiangxi Maternity and Child Healthcare Hospital between January 2016 and June 2017. Informed consent to participate in the study was obtained from the patients. The study was approved by the Ethics Committee of the Jiangxi Maternity and Child Healthcare Hospital. The demographic characteristic of the study group and control group (age, diseases, etc.) were show in Table 1.

\section{Isolation of peripheral blood mononuclear cells}

A total of $8 \mathrm{ml}$ heparinized venous blood were taken from the case and control groups. PBMCs were separated from freshly isolated heparinized venous blood by centrifugation on a Ficoll-Hypaque (Lymphoprep, Sigma, USA) density gradient. Cells at the interface were harvested, washed twice, and resuspended in phosphate buffered saline (PBS). Viable cells were counted by the trypan blue staining method. More than $95 \%$ of the cells were viable.

\section{Treatment protocol}

The four experimental groups included healthy, URPL, high dose of daphnetin-treated PBMCs and low dose of daphnetin-treated PBMCs. Cells were routinely cultured in $75 \mathrm{~cm}^{2}$ tissue culture flasks and incubated in a humidified atmosphere of $5 \% \mathrm{CO}_{2} / 95 \%$ air at $37^{\circ} \mathrm{C}$. PBMCs were treated with high dose of daphnetin $(40 \mu \mathrm{g} / \mathrm{ml})$ or with low dose of daphnetin $(20 \mu \mathrm{g} / \mathrm{ml})$ over a time course of $72 \mathrm{~h}$.

\section{Flow cytometry}

At the end of the experiments, PBMCs were separated from freshly isolated heparinized venous blood and $1 \mathrm{ml}$ of $11 \times 10^{7} / \mathrm{ml}$ of cell suspension was incubated with $10 \mathrm{ng} / \mathrm{ml}$ PMA and $0.5 \mathrm{mM}$ ionomycin (eBioscience, San Diego, CA, USA) for $5 \mathrm{~h}$ at $37^{\circ} \mathrm{C}$ in a $5 \% \mathrm{CO}_{2}$ humidified incubator. Monensin ( $1 \mu \mathrm{l}$ of a $\times 1,000$ solution) (eBioscience) was also applied to enhance intracellular cytokine staining. After incubation, PBMCs were washed in phosphate-buffered saline (PBS) with 009\% (W/V) sodium azide (eBioscience) twice. This was followed by staining with the anti-CD3-FITC and anti-CD8-APC (both from eBioscience), and then incubation for $15 \mathrm{~min}$ at $4{ }^{\circ} \mathrm{C}$. The cells were washed in PBS and fixed with fixation buffer (eBioscience). The cells were washed twice with $1 \mathrm{X}$ permeabilization buffer and incubated with $0.25 \mu \mathrm{g}$ conjugated anti-human IL-17A-PE (both from eBioscience) at room temperature for $20 \mathrm{~min}$. After intracellular staining, the cells were washed with $1 \mathrm{X}$ permeabilization buffer and resuspended in $0.5 \mathrm{ml}$ of permeabilization buffer. The proportion of IL-17-producing T cells in peripheral blood lymphocytes were enumerated by flow cytometry.

To identify Treg cells, PBMCs were stained with $0.25 \mu \mathrm{g}$ FITC-conjugated rabbit monoclonal CD4 anti-body (dilution, 1 : 50; cat. no. 85-11-0048-42) and 0.25 $\mu$ g APC-conjugated rabbit monoclonal CD25 antibody (dilution, $1: 50$; cat. no. 85-17-0259-42) for surface antigens, and $0.25 \mu \mathrm{g}$ PE-conjugated rabbit monoclonal Foxp3 antibody (dilution, 1 : 50; cat. no. 85-12-4776-42), all purchased from eBiosci-

Table 1. The demographic characteristic of the URPL group and healthy group

\begin{tabular}{lcc}
\hline Characteristic & URPL group & Healthy group \\
\hline The number of cases & 35 & 35 \\
\hline Age (years) & $28.5 \pm 4.5$ & $27.6 \pm 4.2$ \\
\hline Have a history of RSA & Yes & No \\
\hline Any other diseases & No & No \\
\hline
\end{tabular}


ence (San Diego, CA, USA), for intracellular molecules, as per the manufacturer's instructions. PBMC $\left(1 \times 10^{6}\right)$ was washed twice in PBS following staining with the fluorochrome-conjugated antibodies specific for cell surface antigen markers for $20 \mathrm{~min}$ in the dark at $4^{\circ}$. In order to stain the intracellular molecule, FoxP3, cells were permeabilized with permeabilization/fixation buffer and stained with anti-FoxP3 antibody following the surface staining. PE-Rat IgG2a was used as an isotype control for anti-FoxP3-PE antibody. Cells were resuspended in $0.5 \mathrm{ml}$ of staining buffer for subsequent flow cytometry analysis. The prepared cells were analyzed on a FACSCalibur flow cytometer (BD Biosciences, Franklin Lakes, NJ, USA). CellQuest Pro software (BD Biosciences) was used for data analysis.

\section{ELISA}

The absorbance of serum interleukin (IL)-6, IL-2 and transforming growth factor (TGF)- $\beta 1$ were detected using the ELISA kit, the concentration was calculated in accordance with the absorbance value.

\section{Quantitative real-time PCR (qRT-PCR)}

The PBMCs were collected in RNase-free tubes and total RNA was extracted using Trizol regent (Invitrogen). RNA samples were quantified with a NanoDrop ${ }^{\mathrm{TM}} 1000$ Spectrophotometer (Thermo scientific) and electrophoresis. Briefly, the peripheral blood cells were homogenized in Trizol reagent, and then chloroform was added to the $1 / 5$ volume of Trizol and mixed thoroughly. The mixture was centrifuged at $12,000 \mathrm{~g}\left(4^{\circ} \mathrm{C}\right)$ for $15 \mathrm{~min}$. The supernatant was collected and mixed with isopropanol at a ratio of $1: 1$ and incubation for $10 \mathrm{~min}$ and was then centrifuged at $14,000 \mathrm{~g}\left(4^{\circ} \mathrm{C}\right)$ for $10 \mathrm{~min}$. The supernatant was removed and $100 \mu 175 \%$ ethanol was added to the tubes and mixed thoroughly. After centrifugation at $8,000 \mathrm{~g}\left(4^{\circ} \mathrm{C}\right)$ for $5 \mathrm{~min}$, the supernatant was removed and the tubes were air-dried. cDNA was synthesized using the prime ScriptTM 1st strand cDNA synthesis kit according to the manufacturer's instructions (Fermentas Thermo). Real-time quantification of target mRNA was performed using a SYBR premix Ex TaqTM II kit (Takara) according to the manufacturer's instructions. Briefly, amplification was performed in a total volume of $10 \mu \mathrm{l}$, and each reaction contained $5 \mu$ l SYBR REmis EX TaqTM, $0.4 \mu$ f forward and reverse PCR primers, $0.2 \mu \mathrm{l}$ ROX reference Dye II, $1 \mu \mathrm{l}$ cDNA and $3 \mu \mathrm{lddH_{2 }} \mathrm{O}$. The real time PCR program consisted of an initial step of $10 \mathrm{~s}$ at $95^{\circ} \mathrm{C}$, followed by 40 cycles of denaturing at $95^{\circ} \mathrm{C}$ for $5 \mathrm{~s}$ and extension at $60^{\circ} \mathrm{C}$ for $31 \mathrm{~s}$. All measurements were performed at least three times. GAPDH was used as a housekeeping gene. The following primer sequences were used: ROR $\gamma \mathrm{t}$-forward, 5-ACC TCA CCG AGG CCA TTC AG-3 and reverse, 5-TAGG CCC GGC ACA TCC TAA C-3; FoxP3-forward, 5-ATC TAC CAC TGG TTC ACA CGC AT-3 and reverse, 5-CTC CAC CCG CAC AAA GCA C-3; GAPDH-forward, 5-GGT GTG AAC CAT GAG AAG TAT GAC A-3 and reverse, 5-GTC CTT CCA CGA TAC CAA AGT TGT-3; STAT3-forward, 5-AAC TCT CAC GGA CGA GGA GCT-3 and reverse, 5-AGT AGT GAA CTG GAC GCC GG-3; STAT5-forward, 5-GCT GGA AGC CTT GCT GAT-3 and reverse, 5-TCC TCA AAC GTC TGG TTG ATC-3.

\section{Statistical analyses}

Quantitative data are expressed as the mean \pm standard deviation (SD). Statistical analyses were performed using the independent samples $t$-test in SPSS soft-ware (SPSS, Inc., Chicago, IL, USA). $P<0.05$ was considered to indicate a statistically significant difference.

\section{Results}

\section{Th17 cells, Treg cells and the ratio of Th17/Treg cells in PBMCs of patients with URPL after treatment of daphnetin}

The percentage of Th17 cells $\left(\mathrm{CD}^{+} \mathrm{CD} 8 \mathrm{IL}-17^{+} \mathrm{T}\right.$ cells) in PBMCs in the healthy group was $0.3 \pm 0.01 \%$, and the percentage of this subset in URPL group was $1.9 \pm 0.07 \%$. After treatment, the high dose group was $0.6 \pm 0.02 \%$ and the low dose group was $0.9 \pm 0.03 \%$. Thus, the percentage of Th17 cells significantly decreased after the treatment of daphnetin ( $p<0.01$, paired t-test, Fig 1A, C). The percentage of Tregs $\left(\mathrm{CD} 4{ }^{+} \mathrm{CD} 25^{+} \mathrm{FoxP} 3^{+} \mathrm{T}\right.$ cells $)$ in healthy group was $4.7 \pm 0.08 \%$, which was $2.3 \pm 0.07 \%$ in URPL group. At the same time, after the treatment of daphnetin, the high dose group was $4.0 \pm 0.06 \%$, while the low dose group was $3.2 \pm 0.07 \%$. Thus, the percentage of Tregs cells significantly decreased after the treatment of daphnetin $(p<0.01$, paired $t$-test, Fig. 1B, C). The mean Th17/Treg ratio in healthy group was $0.06 \pm 0.01$, in URPL group was $0.83 \pm 0.02$ but was $0.15 \pm 0.01$ in high dose group and $0.28 \pm 0.01$ in low dose group. Therefore, the mean Th17/ Treg ratio significantly decreased after the treatment of daphnetin, as compared with URPL group (Fig. 1).

\section{The levels of IL-6, IL-2, TGF- $\beta 1$ and FoxP3, ROR $\gamma$ t, STAT3, STAT5 in four groups}

The concentrations of Th17-type cytokines IL-2 were significantly decreased in the URPL group after the treatment of daphnetin $(p<0.01)$. Treg-type cytokines such as TGF- $\beta 1$ and IL- 6 were significantly increased after treatment $(p<0.01)$. At the same time, daphnetin may induce a decrease in the ratio of ROR $\gamma$ t to Foxp 3 and a Treg cell bias, which would be beneficial for pregnancy maintenance. Futhermore the expression level of STAT3 were 
$\mathbf{A}$

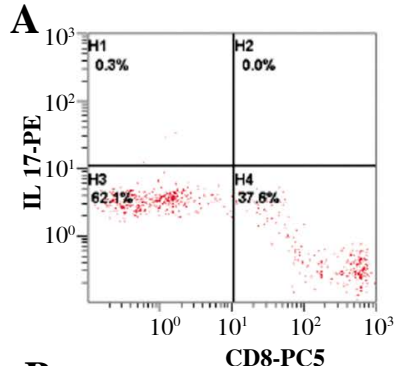

B

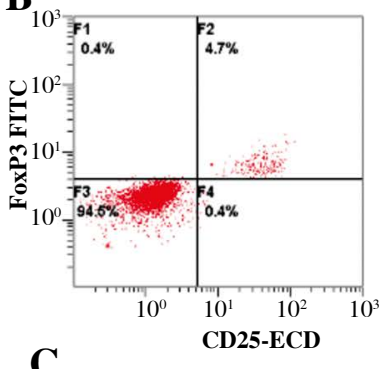

C
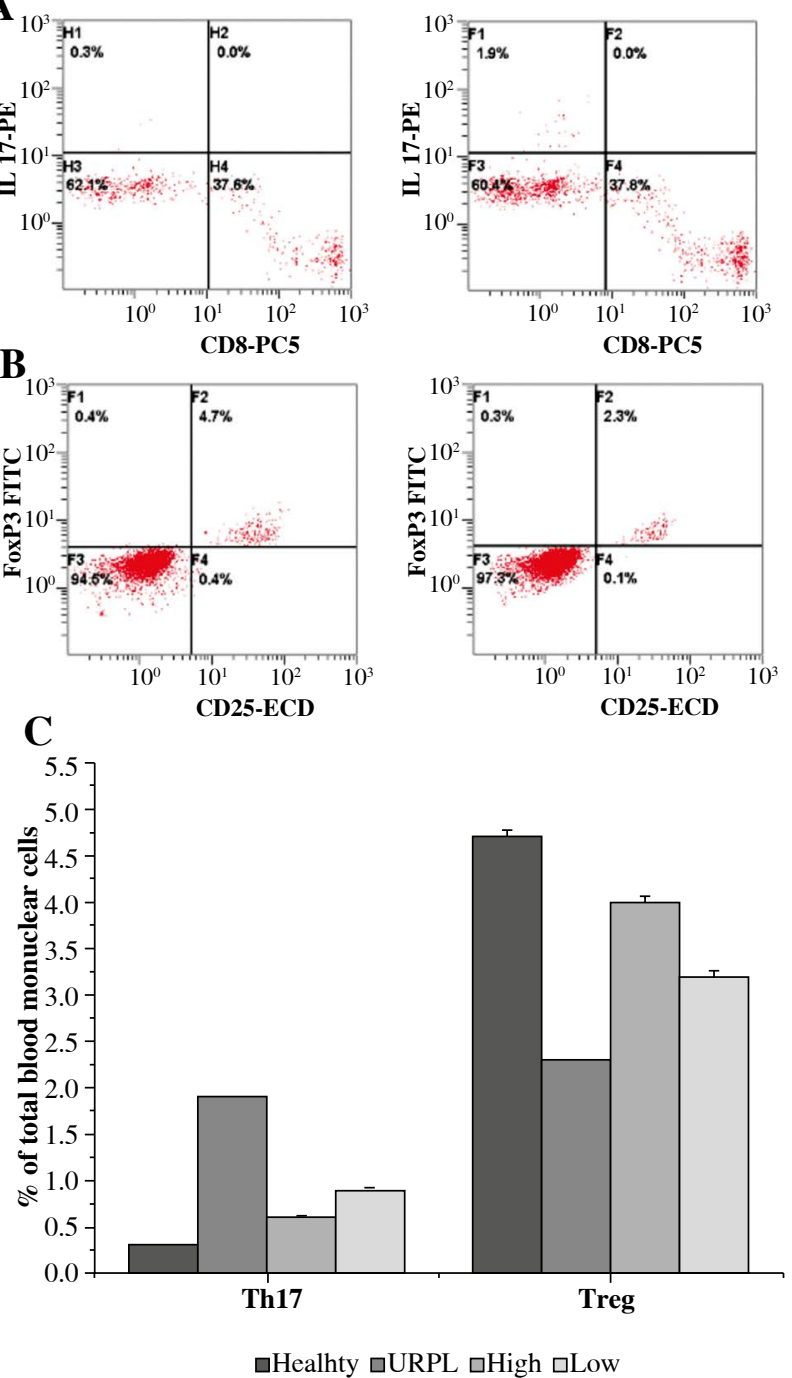
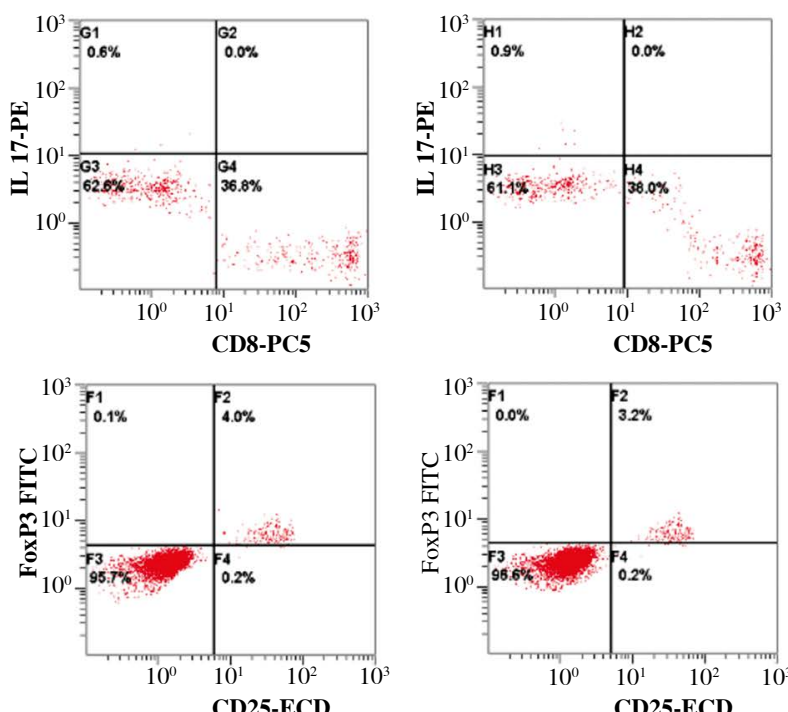

D
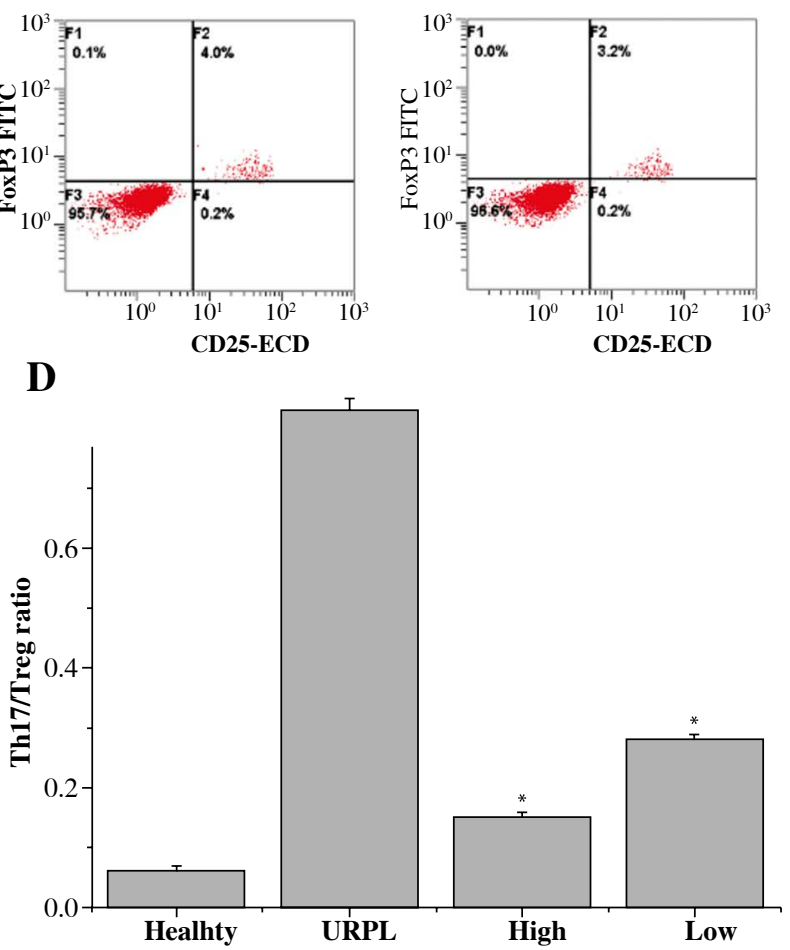

Fig. 1. The percentage of $\mathrm{T}$ helper 17 (Th17) and regulatory $\mathrm{T}$ (Treg) cells in the peripheral blood (PBMCs) of the four experimental groups were detected by flow cytometry. The four experimental groups included health, URPL, high dose of daphnetin-treated PBMCs and low dose of daphnetin-treated PBMCs. Representative CD3+CD8-IL-17A+ flow cytometry plots from the four experimental groups were shown (A) and representative CD4+CD25+FoxP3+ flow cytometry plots from the four experimental groups were shown $(\mathbf{B})$. C) The percentage of Th17 cells in PBMCs significantly decreased after therapy of daphnetin. The percentage of Treg cells in PBMCs significantly increased after treatment $\left({ }^{*} p<0.01\right.$, paired $t$-test). Bar represents the Th17 and Treg cells frequency and the means \pm SEM were indicated. (D) The Th17/ Treg ratio in PBMCs significantly decreased after treatment $(p<0.01$, paired $t$-test). Bar represents the frequency and the means \pm SEM were indicated

higher in the URPL patients whereas STAT5 were lower than those in the control subjects (Fig. 2).

\section{Discussion}

Although the cause of URPL remains unknown, there is increasing evidence that immunologic factors play an important role $[17,18]$. A successful pregnancy relies on the immune tolerance response, which enables the mother to tolerate the semi-allogeneic fetus. Without a delicate maternal-fetal immune balance, a series of pregnancy-related complications will occur, such as spontaneous abortion, preterm birth, and pre-eclampsia. Functional differentiation of T cells, especially the effect of cytokines on URPL, has been one of the focuses of immunological research in recent years [19]. An imbalance in Th17/Treg cells may be involved in autoimmune, inflammatory, and allergic conditions and pregnancy-related complications. Many studies 
A

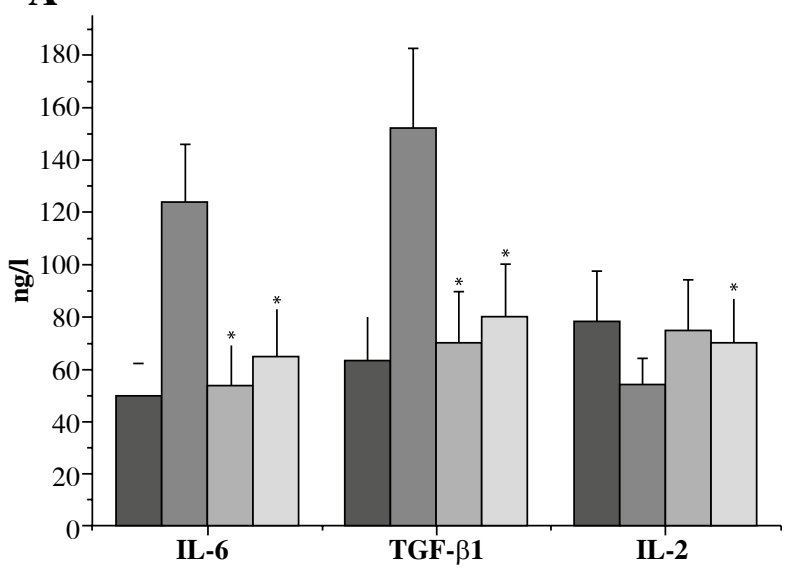

B

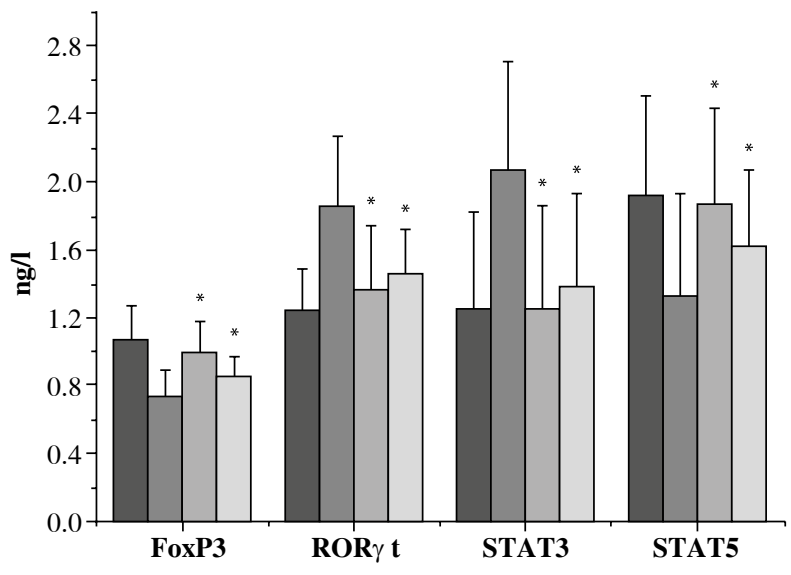

$\square$ Healhty $\square$ URPL $\square$ High $\square$ Low

Fig. 2. The effect of daphnetin on the expression of Th17- and Treg-type related cytokines in the peripheral blood of the four experimental PBMCs groups. The four experimental groups included health, URPL, high dose of E003-treated PBMCs and low dose of daphnetin-treated PBMCs. The level of expression of IL-6, TGF- $\beta 1$ and IL- 2 were detected using ELISA (A) and the level of expression of FoxP3, ROR $\gamma$ t, STAT3 and STAT5 were detected by RT-PCR (B). The concentrations of Th17-type cytokines IL-2 were significantly decreased in the PBMCs of patients with URPL after treatment $(* p<0.01$, paired $t$-test). Treg-type cytokines such as TGF- $\beta 1$ and IL- 6 were significantly increased after treatment $\left({ }^{*} p<0.01\right.$, paired $t$-test). Furthermore, the expression level of STAT3 were higher in the URPL patients' PBMCs whereas STAT5 were lower than those in the control subjects. Values were expressed as the mean \pm SEM

have demonstrated that the ratio of Th17/Treg cells was increased in women with URPL [20-22].

As we all know, there is no specific treatment for URPL. Therapeutic drugs such as glucocorticoids, Aspirin, heparin, active immunotherapy, etc. all have different degrees of side effects. Thus, new drugs with greater efficiency and low toxicity will be highly beneficial to patients with URPL. Daphne marginata is a traditional Chinese medicine possessing the functions of anti-inflammation and odynolysis, in which daphnetin is acknowledged as one of the most effective components of $D$. marginata. Daphnetin has been found having therapeutic effects on autoimmune arthritis through modulating the balance of Tregs and Th17 [23]. In the present study, we first investigated the potential regulatory effect of daphnetin on the balance of Th17 and Treg cells in the PBMCs from patients with URPL. Our data indicated that after treating with daphnetin for $72 \mathrm{~h}$, the balance of Th17/Treg was remarkably improved in PBMCs with URPL. The percentage of Th17 cells significantly decreased, while the percentage of Treg cells were significantly increased after therapy in PBMCs with URPL as compared with the untreated and the healthy group.

In response to various infectious organisms, the adaptive immune system generates distinct responses, which in part are governed by the pattern of cytokines secreted by effector T cells. The cytokines thought to be critical for promoting Th17 differentiation are transforming TGF- $\beta 1$ and IL-6 [24]. In conjunction with T cell receptor stimulation, these cytokines induced the expression of orphan nuclear receptor (ROR $\gamma \mathrm{t}$ ), which is critical for Th17 differentiation [25]. These cytokines activate signal transducers and activators of transcription 3 (STAT3) to direct and maintain Th17 differentiation. Whereas STAT3 appeared to be a key positive regulator of ROR $\gamma \mathrm{t}$, absence of IL-2 or disruption of its signaling by deletion of the transcription factor STAT5 resulted in enhanced Th17 cell development [26]. In this regard, it is notable that daphnetin had a reduction in STAT3 levels resulting in impaired Th17 differentiation and an increase in STAT5 levels resulting in Treg cells differentiation. Furthermore, TGF- $\beta 1$ is also important for maintenance Treg cells. That is, TGF- $\beta 1$ alone promotes expression of the transcription factor FoxP3, whereas the combination of IL- 6 and TGF- $\beta 1$ suppresses FoxP3 expression and enhances IL-17 production. Thus, the differentiation of Treg and Th17 cells by TGF- $\beta 1$ appears to be reciprocally related through the action of IL-6 [27]. In addition, IL-2 also functions to limit the polarization of activated $\mathrm{CD}^{+} \mathrm{T}$ cells into the Th17 lineage [26]. In this study, we found the concentrations of Th17 related molecules IL-2 and STAT3 were significantly decreased in the URPL group after the treatment of daphnetin and Treg related molecules such as TGF- $\beta 1$, IL-6 and STAT5 were significantly increased. This suggests that the treatment of daphnetin is able to inhibit the differentiation of Th17 cells and increase the differentiation of Treg, there- 
by making the Th17/Treg paradigm into the Treg immune bias and thereby promoting the development of full-term pregnancy. Our study is the first to observe the regulatory effect of daphnetin, the active ingredient of traditional Chinese medicine, on Th17 and Treg cells in peripheral blood of patients with URPL. However, this study is limited to clinical specimen studies, and further animal model experiments are needed to observe the mechanism of daphnetin regulating Th17/Treg cell balance in patients with URPL.

\section{Acknowledgments}

We thank everyone who contributed to this study. Medical College of Nanchang University and Jiangxi Maternity and Child Healthcare Hospital supported this research by providing medical information. This work was supported by the Jiangxi natural science foundation (No. 20171BAB215012).

\section{The authors declare no conflict of interest.}

\section{References}

1. Royal College of Obstetricians and Gynaecologists (2011): The Investigation and Treatment of Couples with Recurrent First Trimester and Second Trimester Miscarriage. RCOG, London.

2. Practice Committee of the American Society for Reproductive Medicine (2012): Evaluation and treatment of recurrent pregnancy loss: A committee opinion. Fertil Steril 98: 1103-1111.

3. Little AB (1988): There's many a slip ,twixt implantation and the crib. N Engl J Med 319: 241-242.

4. Wang WJ, Liu FJ, Qu HM, et al. (2013): Regulation of the expression of Th17 cells and regulatory T cells by IL-27 in patients with unexplained early recurrent miscarriage. J Reprod Immunol 99: 39-45.

5. Clark DA (2014): Popular myths in reproductive immunology. J Reprod Immunol 104-105: 54-62.

6. Erlebacher A (2001): Why isn't the fetus rejected? Curr Opin Immunol 13: 590-593.

7. Motedayyen H, Rezaei A, Zarnani AH, et al. (2018): Human amniotic epithelial cells inhibit activation and pro-inflammatory cytokines production of naive CD4+ T cells from women with unexplained recurrent spontaneous abortion. Reprod Biol 18: 182-188.

8. Mei S, Tan J, Chen H, et al. (2010): Changes of CD4+CD25high regulatory $\mathrm{T}$ cells and FOXP3 expression in unexplained recurrent spontaneous abortion patients. Fertil Steril 94: 2244-2247.

9. Raghupathy R (2001): Pregnancy: success and failure within the Th1/Th2/Th3 paradigm. Semin Immunol 13: 219-227.

10. Raghupathy R, Makhseed M, Azizieh F, et al. (1999): Maternal Th1- and Th2-type reactivity to placental antigens in normal human pregnancy and unexplained recurrent spontaneous abortions. Cell Immunol 196: 122-130.

11. Muyayalo KP, Li ZH, Mor G, et al. (2018): Modulatory effect of intravenous immunoglobulin on Th17/Treg cell balance in women with unexplained recurrent spontaneous abortion. Am J Reprod Immunol 80: e13018.
12. Zhu L, Chen H, Liu M, et al. (2017): Treg/Th17 Cell Imbalance and IL- 6 profile in patients with unexplained recurrent spontaneous abortion. Reprod Sci 24: 882-890.

13. Hosseini S, Shokri F, Pour SA, et al. (2016): A shift in the balance of T17 and Treg cells in menstrual blood of women with unexplained recurrent spontaneous abortion. J Reprod Immunol 116: 13-22.

14. Saifi B, Aflatoonian R, Tajik N, et al. (2016): T regulatory markers expression in unexplained recurrent spontaneous abortion. J Matern Fetal Neonatal Med 29: 1175-1180.

15. Gao Q, Shan J, Di L, et al. (2008): Therapeutic effects of daphnetin on adjuvant-induced arthritic rats. J Ethnopharmacol 120: 259-263.

16. Yao R, Fu Y, Li S, et al. (2011): Regulatory effect of daphnetin, a coumarin extracted from Daphne odora, on the balance of Treg and Th17 in collagen-induced arthritis. Eur J Pharmacol 670: 286-294.

17. Zhu LY, Chen X, Xu ZZ, et al. (2015): Changes and clinical significance of peripheral blood helper $\mathrm{T}$ lymphocyte and natural killer (NK) cells in unexplained recurrent spontaneous abortion (URSA) patients after abortion and successful pregnancy. Clin Exp Obstet Gynecol 42: 62-66.

18. Hosseini S, Shokri F, Pour SA, et al. (2018): Diminished frequency of menstrual and peripheral blood NKT-like cells in patients with unexplained recurrent spontaneous abortion and infertile women. Reprod Sci 26: 97-108.

19. Quan X, Yang X (2017): Correlation between unexplained recurrent spontaneous abortion with CD4(+)CD25(+) regulatory T-cell and killer cell immunoglobulin-like receptor levels. Exp Ther Med 14: 1459-1462.

20. Qian J, Zhang N, Lin J, et al. (2018): Distinct pattern of Th17/ Treg cells in pregnant women with a history of unexplained recurrent spontaneous abortion. Biosci Trends 12: 157-167.

21. Roomandeh N, Saremi A, Arasteh J, et al. (2018): Comparing serum levels of Th17 and Treg cytokines in women with unexplained recurrent spontaneous abortion and fertile women. Iran J Immunol 15: 59-67.

22. Sha J, Liu F, Zhai J, et al. (2017): Alteration of Th17 and Foxp3(+) regulatory $\mathrm{T}$ cells in patients with unexplained recurrent spontaneous abortion before and after the therapy of hCG combined with immunoglobulin. Exp Ther Med 14: 1114-1118.

23. Yao R, Fu Y, Li S, et al. (2011): Regulatory effect of daphnetin, a coumarin extracted from Daphne odora, on the balance of Treg and Th17 in collagen-induced arthritis. Eur J Pharmacol 670: 286-294.

24. Mangan PR, Harrington LE, O'Quinn DB, et al. (2006): Transforming growth factor-beta induces development of the $\mathrm{T}(\mathrm{H}) 17$ lineage. Nature 441: 231-234.

25. Ivanov II, McKenzie BS, Zhou L, et al. (2006): The orphan nuclear receptor RORgammat directs the differentiation program of proinflammatory IL-17+ T helper cells. Cell 126: 1121-1133.

26. Laurence A, Tato CM, Davidson TS, et al. (2007): Interleukin-2 signaling via STAT5 constrains T helper 17 cell generation. Immunity 26: 371-381.

27. Bettelli E, Carrier Y, Gao W, et al. (2006): Reciprocal developmental pathways for the generation of pathogenic effector TH17 and regulatory T cells. Nature 441: 235-238. 